\title{
Current Therapy: Defecation Disorders in the Era of Neuromodulation
}

\author{
Lusine Ambartsumyan ${ }^{1}$
}

Accepted: 14 April 2021 / Published online: 18 May 2021

(C) The Author(s), under exclusive licence to Springer Science+Business Media, LLC, part of Springer Nature 2021

Functional defecation disorders such as constipation and fecal incontinence are common in children of all ages. Functional fecal incontinence is subdivided into fecal incontinence associated with stool retention and non-retentive fecal incontinence (FNRFI). Children with FNRFI have normal defecation frequency, absence of stool retention, and normal colonic transit and anorectal and colonic motility parameters [1]. FI has a negative impact on the quality-of-life, social functioning, and emotional well-being of children. Behavioral therapy is the cornerstone of management and focuses on the identification of psychosocial stressors and addressing behavioral and psychological comorbidities. Moreover, education, structured toileting programs, reward systems, and bowel diaries are recommended as first-line conventional therapies $[1,2]$. Biofeedback therapy has not been shown in pediatric studies to provide additional therapeutic benefit when combined with conventional medical therapy for functional constipation, functional retentive fecal incontinence, and FNRFI [3, 4]. However, in a recent randomized controlled trial of children with FNRFI, Abdelrahman et al. compared the efficacy of biofeedback therapy, transcutaneous posterior tibial nerve stimulation (TPTNS) and Kegal exercises + diet at 3- and 6-months post-intervention. Significant improvement was seen in all three groups pre- and post-intervention at 3 and 6 months of follow-up. Children who received biofeedback had the highest significant decrease in FI. score when compared with the other groups [5].

The application of neuromodulation in the treatment of defecation disorders in children has gained popularity over the last few decades. Invasive and noninvasive modalities include implantable or transcutaneous sacral nerve stimulators (SNS), percutaneous or transcutaneous posterior tibial nerve stimulators (PTNS), and interferential (IF) therapy.

Lusine Ambartsumyan

lusine.ambartsumyan@seattlechildrens.org

1 Division of Gastroenterology \& Hepatology, Seattle Children's Hospital, 4800 Sand Point Way NE, Seattle, WA 98105, USA
Studies in children exploring the above modalities for functional and organic fecal incontinence and constipation are limited and inundated with limitations. Thus, its applicability as a first-line treatment modality for defecation disorders in children remains to be further elucidated. To date, there are no pediatric randomized controlled trials evaluating the efficacy of SNS in the treatment of functional and organic constipation and fecal incontinence. In children, implantable SNS has been shown to be promising in decreasing the use of laxatives and antegrade continence enemas, decreasing fecal incontinence frequency, improving abdominal pain, and increasing the quality-of-life scores [6]. Nevertheless, loss of efficacy requiring surgical intervention and post-implant complications were reported in nearly $38 \%$ of pooled patients, constituting major limitations [6]. Consequently, less invasive modalities, especially as adjunctive treatment to conventional medical therapy, improve fecal incontinence and bowel movement frequency in children without the associated complications seen with invasive SNS [6].

Studies in children of the efficacy of neuromodulation are limited by a heterogenous patient population (e.g., anorectal malformations, neurogenic bowel, Hirschsprung disease), a lack of randomization, small sample size, variable length of follow-up, and lack of a sham control. In a systematic review and meta-analysis, Tan et al. demonstrated that sham stimulation is associated with statistically significant improvements in quality-of-life scores and in clinical symptoms of fecal incontinence and constipation [7]. In a single-center, double-blind randomized clinical study, Sharifi-Rad et al. compared the outcomes of children with functional constipation who underwent IF electrical stimulation and pelvic floor muscle (PFM) exercises to those who received sham stimulation and PFM exercises [8]. Patients who received IF electrical stimulation had significantly superior clinical improvement than the sham stimulation group. This study highlighted the utility of IF electrical stimulation as an adjunct therapy, but it also demonstrated the feasibility of sham-controlled trials in noninvasive neuromodulation techniques [8]. 
In this issue of Digestive Diseases and Sciences, LadiSeyedian et al. [9] present a prospective, single-center randomized clinical trial comparing the effect of transcutaneous functional electrical stimulation (TFES) combined with biofeedback with TFES alone in children with NRFI. This study demonstrates that TFES alone and in combination with biofeedback improved fecal incontinence, abdominal pain, and quality-of-life scores. No differences in the aforementioned parameters were noted between the two groups, illustrating the efficacy of TFES as an adjunctive therapy in the treatment of refractory FNRFI.

Transcutaneous electrical stimulation (TES) and functional electrical stimulation (FES) are a form of noninvasive surface electrical stimulation in which the electrical current produces a skeletal muscle contraction [10]. In the present study, two electrodes were placed on the gluteal muscles with the intensity adjusted to produce muscle contraction, precluding a sham control within the study and predisposing the study to a sham placebo effect. The lack of complications offered by this noninvasive modality is a significant benefit when compared with its invasive counterparts and thus more appropriate and palatable for the pediatric population.

Improvements in clinical parameters and quality-of-life by TFES as demonstrated in the study were maintained 6 months after treatment. While this is mostly reassuring, indicating likely long-term effectiveness, it would be of benefit to have longer-term prospective data in order to truly demonstrate the utility of this intervention. The noninvasive methods are advantageous since they can be easily re-introduced and may serve as adjunctive therapy. Well-designed clinical trials are necessary to investigate the effectiveness of noninvasive neuromodulation in the setting of adjunctive therapies (pelvic floor therapy, conventional medical treatment, biofeedback, behavioral therapy) over long-term follow-up.

A strength of the current study is that all children met the diagnostic Rome IV criteria for FNRFI and had failed conventional therapy, in contrast to most published pediatric studies that study heterogenous populations comprised of a mix of functional and organic defecation disorders. Such variable pathophysiological pathways of fecal incontinence complicate the accurate identification of the mechanism of action of the intervention. In their systematic review, Carrington et al. demonstrated that SNS likely influences anorectal function at a pelvic afferent or central level indicating alterations of sensory rather than motor pathways [11]. Children with FNRFI are thought to have normal anorectal and colonic motility parameters. Therefore, neuromodulation of pelvic floor sensory and cortical pathways may explain the benefits demonstrated by TFES in children with FNRFI.

As pediatric studies utilizing neuromodulation pick up momentum, further clarification of the contribution of neuromodulation toward the treatment of defecation disorders is needed. Meticulous study design and multicenter collaborative efforts should be undertaken in order to overcome the aforementioned limitations of the pediatric studies. Efforts should be made to identify those who will benefit from this modality and to determine the clinical and diagnostic predictors of success. Similar to the surgical paradigm, the future of neuromodulation is minimally invasive modalities that offer the most innovative disease-specific therapeutic solutions. Collaboration between centers and national databases of neuromodulation outcomes would allow us to use big data and create therapeutic algorithms.

\section{Declarations}

Conflict of interest Consultant for Takeda Pharmaceuticals.

\section{References}

1. Hyams JS, Di Lorenzo C, Saps M, Shulman RJ, Staiano A, van Tilburg M. Childhood functional gastrointestinal disorders: child/adolescent. Gastroenterology 2016;150:1456.

2. Koppen IJ, von Gontard A, Chase J, Cooper CS, Rittig CS, Bauer SB et al. Management of functional nonretentive fecal incontinence in children: recommendations from the international children's continence society. J Pediatr Urol. 2016;12:56-64.

3. Rao SS, Benninga MA, Bharucha AE, Chiarioni G, Di Lorenzo C, Whitehead WE. ANMS-ESNM position paper and consensus guidelines on biofeedback therapy for anorectal disorders. Neurogastroenterol Motil. 2015;27:594-609.

4. van der Plas RN, Benninga MA, Redekop WK, Taminiau JA, Buller HA. Randomised trial of biofeedback training for encopresis. Arch Dis Child. 1996;75:367-374.

5. Abdelrahman EM, Abdel Ghafar MA, Selim AO, Ali OI, Balbaa MA. Biofeedback versus bilateral transcutaneous posterior tibial nerve stimulation in the treatment of functional non-retentive fecal incontinence in children: a randomized controlled trial. $J$ Pediatr Surg. 2020.

6. Iacona R, Ramage L, Malakounides G. Current state of neuromodulation for constipation and fecal incontinence in children: a systematic review. Eur J Pediatr Surg. 2019;29:495-503.

7. Tan K, Wells CI, Dinning P, Bissett IP, O'Grady G. Placebo response rates in electrical nerve stimulation trials for fecal incontinence and constipation: a systematic review and metaanalysis. Neuromodulation. 2020;23:1108-1116.

8. Sharifi-Rad L, Ladi-Seyedian SS, Manouchehri N, Alimadadi H, Allahverdi B, Motamed F et al. Effects of interferential electrical stimulation plus pelvic floor muscles exercises on functional constipation in children: a randomized clinical trial. Am J Gastroenterol. 2018;113:295-302.

9. Ladi-Seyedian SS, Sharifi-Rad L, Alimadadi H, Nabavizadeh B, Manouchehri N, Allahverdi B et al. Comparative efficacy of transcutaneous functional electrical stimulation with or without biofeedback therapy on functional non-retentive fecal incontinence in children: A randomized clinical trial. 
Dig Dis Sci. (Epub ahead of print). https://doi.org/10.1007/ s10620-021-07012-3.

10. Southwell BR. Electro-neuromodulation for colonic disordersreview of meta-analyses, systematic reviews, and RCTs. Neuromodulation. 2020;23:1061-1081.

11. Carrington EV, Evers J, Grossi U, Dinning PG, Scott SM, O'Connell PR et al. A systematic review of sacral nerve stimulation mechanisms in the treatment of fecal incontinence and constipation. Neurogastroenterol Motil. 2014;26:1222-1237.
Publisher's Note Springer Nature remains neutral with regard to jurisdictional claims in published maps and institutional affiliations. 